\title{
EFFECT OF ABSORPTION IN DIFFRACTION PROFILES IN THE MIXING OF LaB6 AND CeO2
}

\author{
Batista, A. M. L. ${ }^{1 *}$; Lima, A. N. C. ${ }^{1}$; Muniz, F. T. L. ${ }^{2}$, Sasaki, J. M. ${ }^{2}$ \\ ${ }^{1}$ Departamento de Engenharia Metalúrgica, UFC, Fortaleza, Ceará, Brasil \\ 2 Departamento de Física, UFC, Fortaleza, Ceará, Brasil \\ *andersondmlb@gmail.com
}

The absorption effect in the materials, in the X-ray diffraction, is one factor that attenuates the intensity of the diffraction profiles. Samples that show particles in average sizes of the order of micrometers, are generally more absorbers, because they have a larger thickness than nanometer scale. When these particles reach size of this order of magnitude, the kinematic theory take place the dynamic theory of X-ray diffraction. In this work, a mixture of lanthanum hexaboride (SRM 660b) and cerium oxide crystals with a mass fraction of $50 \%$ for each compound was prepared for X-ray powder diffraction measurements. The objective of this study is to analyze the effect of absorption (xxx, yyy) in the diffraction profile of this mixture. The materials have high degree of homogeneity, low microstrain and particle sizes of the order of micrometers. It is expected that after a careful analysis, it can shows changing in diffraction peak intensities. The use of software that still is in development, based on the kinematic theory and dynamical theory, gives reliability to the results presented in this paper. However, the asymmetry in the profiles should be provided only by the software based on the Theory Dynamic, being this, the theory that considers these phenomena related to thick particles. Finally, after the comparison of the observed and simulated profiles, it is expected be possible to detect the effects dynamics which the mixture is subjected. 